erhoben, sondern der Weg, nämlich die stetige Reduktion des Stoffflußvolumens, wird als pauschale Vorsorgestrategie zum relevanten Ziel erklärt. Die Ausschöpfung eines behaupteten Reduktionsspielraums - etwa im Rahmen einer Faktor 10-, Faktor 5- oder Faktor 4-Gesellschaft wird zum Leitbild einer am Nachhaltigkeitspostulat ausgerichteten Umweltpolitik.

\section{Internalisierungsstrategie versus umfassende Lenkungsaufgabe}

Um nicht falsch verstanden zu werden: Auch die Nachhaltigkeitsinterpretationen im Sinne des ökologischen Ein-Säulen- oder des Drei-SäulenModells werden, vor allem wenn alle Länder dieser Welt die Produktions- und Konsummuster der entwickelten Industrienationen kopieren wollen, zwecks Verhinderung von Zuständen von Nichtnachhaltigkeit mít einer Reduktion der Materialintensität, einer verstärkten Kreislaufführung der Stoffe, einer Steigerung der Langlebigkeit vieler Produkte oder mit gravierenden Änderungen der Konsummuster verbunden sein. Dies gilt aber nur solange, wie die oben angesprochenen Leitplanken, die unter ökonomischen Aspekten Grenzen darstellen, ab denen die Grenzschadenskosten die Grenzvermeidungskosten überschreiten, durchbrochen werden und die Gefahr einer nicht nachhaltigen Entwicklung besteht. Dies ist aber etwas anderes als - losgelöst von den ökonomischen Implikationen - die Überstrapazierung des Vorsorgeprinzips im Sinne des Forderns steter Reduktion des Stoffflußvolumens. Eine solche stoffpolitische Forderung nach kontinuierlicher Reduktion des Stoffflußvolumens ist auch mehr als eine Internalisierungsstrategie; sie ist eine umfassende Lenkungsaufgabe, bei der die Akteure des Wirtschaftssystems über eine sukzessiv steigende Ressourcenbesteuerung mit der staatlich geführten Preispeitsche zur Effizienz- oder Suffizienzrevolution getrieben werden sollen. Eine solche Konzeption kann man nicht mehr als ökonomieveträglich oder marktwirtschaftskonform kennzeichnen.

Dies gilt auch für jene Spielformen des Reduktionskonzepts, die als zu berücksichtigende $\mathrm{Ne}$ benbedingung die Makroneutralität - keine nachteiligen Nebenwirkungen auf Beschäftigung, Preisniveau oder wirtschaftliches Wachstum fordern (5) oder sogar behaupten, eine solche Politik sei wohlstandssteigernder Natur - eine Art makroökonomische Doppeldividende (mehr Umweltschutz mit mehr Wohlstand). Hier mischen sich normative Überlegungen - Verteilungsziele und Hochgewichtung des Vorsorgeprinzips - mit schwer nachweisbaren Behauptungen bezüglich der Makroeffekte einer Politik immer stärkerer Staatsdeterminiertheit der Ressourcenpreise, insbesondere wenn dies im natio- nalen Alleingang geschieht. Solche radikalen Forderungen bestimmter Vertreter der ökologischen Ökonomie stellen eine Transformation unseres Wirtschaftssystems dar und stehen, was ihre Makroneutralität oder ökonomische Vorteilhaftigkeit betrifft, auf schwachen Fundamenten.

Paul Klemmer, Essen

\section{Anmerkungen}

1) Vgl. Enquete-Kommission »Schutz des Menschen und der Umwelt « des Deutschen Bundestages (Hrsg. ): Die Industriegesellschaft gestalten. Perspektiven für einen nachhaltigen Umgang mit Stoff- und Materialströmen, Endbericht, Bonn 1994 (gleichzeitig als Bundestagsdrucksache 12/8260) erschienen).

2) So wurden vom Bundesminister für Wirtschaft 1995 einige Forschungsaufträge vergeben, die sich mit den ordnungspolitischen Implikationen des Nachhaltigkeitspostulàts befassen, wobei zwangsläufig auf die Forderung nach einer umfassenden Stoffpolitik eingegangen werden muß.

3) Vgl. zum nachfolgenden auch Klemmer, P.: Nachhaltige Entwicklung - aus ökonomischer Sicht, Zeitschrift für angewandte Umweltforschung, 7. Jg., 1994, S. $14 \mathrm{ff}$.

4) Vgl. etwa Enquete-Kommission »Schutz des Menschen und der Umwelt « des Deutschen Bundestages (Hrsg. ): a.a. O., S. 32.

5) So z. B. die Enquete-Kommission »Schutz des Menschen und der Umwelt« des. Deutschen Bundestages (Hrsg. ): a.a. O., S. 49.

\title{
Monetäre Dynamik und Nachhaltigkeit
}

Der Begriff der »Nachhaltigkeit« bzw. »Sustainable Development « ist seit der Veröffentlichung des Brundtland-Berichtes im Jahre 1987 zu der wahrscheinlich am häufigsten gebrauchten Vokabel in der Oekonomie geworden, wenn es darum geht, ökologische Ziele für die Zukunft zu formulieren. In diesem Zusammenhang hat man sich bis heute aber nur wenig mit der längerfristigen Dynamik heutiger Wirtschaftssysteme beschäftigt. Es wird kaum danach gefragt, welche Logik hinter den wirtschaftlichen Vorgängen steckt und welche Konsequenzen sich daraus für Wirtschaft und Umwelt ergeben. Erst wenn man sich aber mit diesen Themen beschäftigt wird man auch Antworten auf nicht ganz unerhebliche Fragen finden, wie:

- Besteht im heutigen Weltwirtschaftssystem ein Zwang zum Wachstum oder ist auch eine quantitativ nicht mehr wachsende Wirtschaft möglich?

- Lässt sich Wirtschaftswachstum mit nachhaltiger Entwicklung vereinbaren, d. h., kann es ein nachhaltiges Wachstum überhaupt geben? Im folgenden versucht dieser Beitrag, zumindest einige Aspekte der wirtschaftlichen Wachstumsdynamik zu beschreiben, um so einer Antwort auf die eben gestellten Fragen näherzukommen.

\section{Monetäre Dynamik und Wachstumszwang}

Im Grunde genommen geht es in einer Geldwirtschaft, so wie sie in den heutigen Industrieländern schon seit hunderten von Jahren existieren, nur um eines: nämlich aus einer bestimmten Geldsumme M (money) eine höhere Geldsumme M' zu machen. Dies ist der entscheidende Grund, weshalb ein Unternehmen überhaupt produziert. Unternehmen produzieren, weil sie damit rechnen, durch den Verkauf von Gütern bzw. Dienstleistungen im Verlauf der Zeit eine höhere Geldmenge $\mathrm{M}$ ' einzunehmen als sie ursprünglich für die Bezahlung der Produktionsfaktoren Arbeit (Löhne) und Kapital (Investitionen) ausgegeben haben, um so einen Gewinn $\mathrm{G}=\mathrm{M}$ '-M zu realisieren (1). Dies ist, ganz einfach ausgedrückt, die fundamentale Logik einer Geldwirtschaft, welche diese zu einer ständigen Expansion antreibt (2).

Der wirtschaftliche Kreislauf ist somit für das Unternehmen ein kontinuierlicher Prozess, der sich in einer auf Marx (und nicht auf den Marxismus!) zurückgehenden Terminologie als M-CM'-Kreislauf (Geld-Ware-Geld-Kreislauf) beschreiben lässt. Der Kreislauf beginnt damit, dass ein Unternehmen eine bestimmte Geldsumme M investiert, um damit die für die Produktion notwendigen Produktionsfaktoren Arbeit und Kapital zu bezahlen. Erst an zweiter Stelle kommen reale, d. h. physische Güter C (commodities) hin$\mathrm{zu}$, indem die Unternehmen Anlagen und Maschinen kaufen (Realkapital), um damit Güter und Dienstleistungen zu produzieren. Durch den Verkauf der mit Hilfe von Arbeit und Kapital hergestellten Güter verwandelt das Unternehmen schliesslich die produzierten Güter und Dienstleistungen wieder in Geld (M'), wobei gilt, dass die erwartete Geldmenge M' grösser sein muss als die ursprünglich investierte Geldmenge $M$. Die in dem Wirtschaftskreislauf zirkulierende Geldmenge vergrössert sich somit von Periode zu Periode, solange sich die Gewinnerwartungen tatsächlich erfüllen.

Handeln alle Unternehmen in einer Wirtschaft nach dem Prinzip des M-C-M'-Kreislaufs, so wächst auch die gesamte Wirtschaft, sofern die erwarteten Einnahmen M' sich bei einer Mehrheit der Unternehmen auch tatsächlich einstellen. Solange der M-C-M'-Kreislauf funktioniert, funktioniert auch die Wirtschaft. Dank des Wachstums ist die Wirtschaft kein Nullsummenspiel, sondern ermöglicht es den Wirtschaftsakteuren, einen Gewinn zu machen bzw. das Einkommen zu erhöhen, ohne dass sich dadurch der Gewinn bei andern Wirtschaftsakteuren bzw. das Einkommen verringern muss, wie dies bei einem Nullsummenspiel der Fall wäre. Das ist ein we- 
sentlicher Grund dafür, warum Wachstum auch in Zusammenhang mit der Entwicklung der dritten Welt immer so stark propagiert wird. Es ermöglicht den Entwicklungsländern eine Erhöhung des Wohlstandes, ohne dass wir in den Industrieländern dafür auf irgend etwas verzichten müssten.

\section{Die Bedeutung der Finanzmärkte}

In der heutigen Wirtschaft ergibt sich der Wert aller Investitionen in Realkapital (Maschinen und Anlagen) letztlich auf Finanzmärkten. Die Motivation hinter allen Investitionen sind erwartete Gewinne, die sich wiederum auf die Preise von Wertpapieren wie Aktien und Obligationen auswirken, und dadurch das Vermögen der Investoren erhöhen (3). Diese Transformation von erwarteten zukünftigen Erträgen in Wertpapierpreise ist notwendig, um potentiellen Investoren einen Anreiz zu geben, in das betreffende Unternehmen zu investieren. Erwartet man, dass ein bestimmtes Unternehmen in Zukunft erfolgreich sein wird, so werden sich die Preise der Wertpapiere des betreffenden Unternehmens erhöhen, und können dann mit Gewinn wieder verkauft werden. Alle erwarteten zukünftigen Erträge, die Unternehmen durch ihre produktive Tätigkeit erwirtschaften, kommen also auf Finanzmärkten zum Ausdruck, wo die produktiven Tätigkeiten in Geld bewertet werden.

Wenn nun der Gegenwartswert einer Investition von den abdiskontierten zukünftigen Gewinnen abhängt und wenn diese wiederum die Preise der Wertpapiere bestimmen, dann wirken sich die Erwartungen über zukünftiges Wachstum auf die heutigen Preise der Wertpapiere aus (4). Eine Aenderung dieser Erwartungen führt in diesem Fall zu unmittelbaren Aenderungen der Wertpapierpreise. Erwarteten die Wirtschaftsakteure beispielsweise ein bestimmtes Wachstum der zukünftigen Gewinne und ändern sich diese Erwartungen in der Weise, dass mit keinem zukünftigen Wachstum mehr gerechnet wird, dann führt dies zu einem Preisverfall auf den entsprechenden Finanzmärkten. Das bedeutet, dass die Erwartung eines zukünftigen Nullwachstums oder bereits eines geringer werdenden Wachstums das gegenwärtige Finanzvermögen vermindert, da die Preise, zu welchen die Wertpapiere wieder verkauft werden können, sinken. Für die Investoren bringt dies reale Verluste, wie dies bei Börsencrashs der Fall war.

Die Abhängigkeit des gegenwärtigen Vermögens einer Volkswirtschaft von zukünftigem Wachstum ist ein wesentlicher Grund dafür, weshalb sich heutige Geldwirtschaftssysteme in einem effektiven Wachstumszwang befinden. Durch frühere Wachstumserwartungen wurde unser heutiges Vermögen dermassen in die Höhe katapultiert, dass wir dazu verdammt sind, diese Wachstumserwartungen auch für die Zukunft aufrechtzuerhalten. Andernfalls bricht unser gesamtes auf fiktiven zukünftigen Gewinnen beruhendes Vermögen zusammen. Und Wachstumserwartungen lassen sich auf die Dauer nur auf- rechterhalten, wenn dieses Wachstum auch eintritt. Dies ist schon deshalb der Fall, weil die Unternehmen jedes Jahr Zinsen und Dividenden für das erhaltene Geldkapital bezahlen müssen, wozu sie über längere Zeit nur in der Lage sind, wenn das erwartete Wachstum auch tatsächlich eintrifft.

Diese Ueberlegungen treffen sowohl für die gesamte Volkswirtschaft wie auch für das einzelne Unternehmen zu. Ist damit zu rechnen, dass in einem bestimmten Unternehmen in Zukunft kein Wachstum mehr zu verzeichnen sein wird, dann werden die Investoren die Wertpapiere des betreffenden Unternehmens möglichst schnell verkaufen, um in ein erfolgreicheres Unternehmen zu investieren. Dadurch kommt es zu einem Preisverfall der Wertpapiere des entsprechenden Unternehmens, dem es dann kaum mehr gelingen wird, neue Investoren zu finden. Dies ist nur möglich, wenn es das Unternehmen schafft, die Erwartungen für ein zukünftiges Wachstum wieder zu beleben. Für das einzelne Unternehmen ist Wachstum somit eine Notwendigkeit, um Investoren zu finden, die bereit sind, Geldkapital zu investieren. Gelingt dies nicht, so verschwindet es mit der Zeit vom Markt. Volkswirtschaftlich ist dies natürlich noch kein Problem, solange es noch Unternehmen gibt, bei denen ein Wachstum erwartet wird. Erst wenn die ganze Wirtschaft in eine depressive Stimmung gerät und allgemein mit keinem Wachstum mehr gerechnet wird, dann kommt es zu einem Crash und zu einem allgemeinen Vermögensverlust. Und da sich Finanzmärkte in immer schnellerem Tempo entwickeln und ein immer grösseres Vermögen auf zukünftigen Erwartungen aufbaut, wird auch der Zwang zum Wachstum mit zunehmendem Wohlstand nicht etwa geringer sondern grösser.

Führt Wachstum auch zu einer Zunahme von Naturverbrauch und Umweltbelastungen?

Das vorherige Kapitel versuchte aufzuzeigen, warum in den modernen Geldwirtschaftssystemen, so wie sie bis heute funktionieren, auf Wachstum nicht verzichtet werden kann. Damit ist aber noch nichts darüber ausgesagt, ob Wachstum auch zwingend zu einer Zunahme von Naturverbrauch und Umweltbelastungen führt, oder, in bezug auf unser Thema, ob sich Wachstum grundsätzlich mit nachhaltiger Entwicklung vereinbaren lässt. Die Meinungen dazu sind durchaus kontrovers. Der bekannte amerikanische Umweltökonom Hermann Daly bezeichnet ein nachhaltiges Wachstum als einen Widerspruch in sich, wenn er schreibt:

"Since the human economy is a subsystem of a finite global ecosystem which does not grow, even though it does develop, it is clear that growth of the economy cannot be sustainable over long periods of time. The term »sustainable growth « should be rejected as a bad oxymoron « (5).

Die Kommission der Europäischen Gemeinschaften spricht hingegen ganz locker von »...employment creating and environmentally sustainable economic growth « (6). Kann es also ein nachhaltiges Wachstum geben?

Der gesamte technische Fortschritt seit der industriellen Revolution ist dadurch gekennzeichnet, dass die immer teurer werdende Arbeit, deren Entlöhnung heute den grössten Teil der Wertschöpfung ausmacht, durch Maschinen (Realkapital) ersetzt wurde, die mit immer billiger werdender Energie angetrieben wurden und immer billiger werdende Rohstoffe verarbeiteten (7). So verschwanden die Arbeiter durch Automatisierung zunehmend aus den Fabrikhallen und übernahmen stattdessen Schreibtisch-Jobs. Nun lohnt sich dieser arbeitssparende technische Fortschritt in der Produktion aber vielfach nur, wenn es auch zu einer Zunahme der produzierten Menge kommt. Ein Wachstum der wirtschaftlichen Wertschöpfung (Wirtschaftswachstum) führt somit tendenziell auch zu steigenden Produktionsmengen in der Industrie, was zu zunehmendem Naturverbrauch und Umweltbelastungen führt. Nachhaltiges Wachstum scheint aus dieser Perspektive also tatsächlich unmöglich zu sein. Nun lassen sich zunehmender Naturverbrauch und Umweltbelastungen aber gemäss konventioneller ökonomischer Logik durch staatliche Massnahmen wie Steuern oder Abgaben vermeiden, die dafür sorgen, dass die Umwelt in das Marktpreissystem integriert wird. Dies würde zu einer Verteuerung von Energie und Rohstoffen führen (höhere variable Kosten), wodurch deren Verbrauch zurückgehen sollte. Insbesondere würde sich Energie auch gegenüber der Arbeit verteuern, die damit auch in der Produktion wieder konkurrenzfähiger würde. Ein Vorschlag, der ganz gezielt in diese Richtung geht, ist die Idee eines ökologischen Umbaus des Steuersystems (8), der nicht nur eine Energiesteuer propagiert, sondern gleichzeitig eine Entsteuerung der Arbeit verlangt, so dass insgesamt keine höhere Steuerbelastung resultiert. Inwieweit das tatsächlich klappt, wird sich hoffentlich bald einmal zeigen, wenn das erste Land eine solche ökologische Steuerreform einführt. Eines ist jedenfalls sicher: Erst wenn die Wirtschaftsakteure wissen, dass sich die Leistungen der Umwelt in Zukunft verteuern werden, werden sie bereit sein, Technologien und Produkte zu fördern, die mit geringerem Naturverbrauch und Umweltbelastungen verbunden sind.

\section{Fazit}

In diesem Beitrag wurde versucht, dem ökonomischen Gehalt des Nachhaltigkeitsbegriffs etwas auf die Spur zu kommen und nachhaltige Naturnutzung in Zusammenhang mit der wirtschaftlichen Dynamik heutiger Industriewirtschaften zu diskutieren. Dabei standen vor allem zwei Fragen im Mittelpunkt, die sich jetzt zumindest ansatzweise beantworten lassen. Diese Fragen lauteten:

Frage: Besteht im heutigen Weltwirtschaftssystem ein Zwang zum Wachstum oder ist auch eine quantitativ nicht mehr wachsende Wirtschaft möglich? 
Anwort: Es besteht ein Wachstumszwang, da wir es heute mit Geldwirtschaften zu tun haben, in welchen das heutige Geldvermögen von zukünftigen Wachstumserwartungen abhängt. Eine Abkehr vom Wirtschaftswachstum ist somit ohne grundlegende Aenderung der Wirtschaftssysteme nicht möglich.

Frage: Lässt sich Wirtschaftswachstum mit nachhaltiger Entwicklung vereinbaren, d.h. kann es ein nachhaltiges Wachstum überhaupt geben? Antwort: Vielleicht ist dies in Zukunft möglich. Bisher hat es jedoch kein nachhaltiges Wachstum gegeben. Allerdings weiss man noch zu wenig darüber, ob durch staatliche Massnahmen ein solches Wachstum in Zukunft eingeleitet werden kann, da gleichzeitig eine Tendenz besteht, die Produktionsmengen wegen skalenerhöhendem technischen Fortschritt laufend zu erhöhen. Energiesteuern und andere ökologisch motivierten Korrekturen des Marktpreissystems wurden bisher, obwohl seit Jahrzehnten von Oekonomen vorgeschlagen, in der Praxis noch kaum in nennenswerter Höhe eingeführt.

Mathias Binswanger, St. Gallen
Anmerkungen

1) Selbstverständlich müssen, wie wir weiter unten noch sehen werden, alle erwarteten zukünftigen Geldströme abdiskontiert werden

2) siehe z. B. Heilbronner, 1986.

3) siehe beispielsweise Minsky, 1986, S. $348 \mathrm{f}$

4) ausführlicher beschrieben ist dies in Binswanger, 1994, 1995

5) Daly, 1992,

6) Kommission der europäischen Gemeinschaften, 1993

7) siehe z. B. Opschoor, 1991, S. 35

8) siehe z.B. v. Weizsäcker/Jeninghaus/Mauch/Iten, 1992

\section{Literatur}

- Binswanger, M. 1994: Wirtschaftswachstum durch »Profits without Production «? In: Binswanger, H. C. / von Flotow (Hg. ): Geld und Wachstum, Stuttgart, S. 161-184.

- Binswanger, M. 1995: Sustainable Development: Utopie in einer wachsenden Wirtschaft?, in: Zeitschrift für Umweltpolitik \& Umweltrecht 1, S 1-19

- Christensen, P. 1991: Driving Forces, Increasing Returns and Ecological Sustainability, in Constanza, R. (editor): Ecological Economics, New York

- Daly, H. 1992: Steady State Economics, London

- El Serafy, S. 1992: Oekologische Tragfähgigkeit, Einkommensmessung und Wachstum, in
Goodland et al.: Nach dem Brundtland-Bericht: Umweltverträgliche wirtschaftliche Entwicklung, UNESCO, Bonn

- Hauff, V. (Hg. ) 1987: Unsere gemeinsame Zukunft. Der Brundtland-Bericht der Weltkommission für Umwelt und Entwicklung, Greven

- Heilbronner, R. 1986: The Nature and Logic of Capitalism, New York

- Kommission der europäischen Gemeinschaften 1993: Economic Growth and Environmental Sustainability: a strategic view for the Community. Working Paper of the Commission services, Brüssel

- Minsky, H. 1986: »The Evolution of Financial Institutions and the Performance of the Economy.» Journal of Economic Issues 20, (June): 345-53.

- OECD 1993: Environmental Data, Paris

- Opschoor, H. 1992: Sustainable Development, The Economic Process and Economic Analysis, in: Opschoor, H. (Hg. ): Environment, Economy and Sustainable Development, Amsterdam

- Pearce/Turner 1990: Economics of Natural Resources and the Environment, London

- von Weizsäcker / Jesinghaus / Mauch / Iten 1992: Ökologische Steuerreform, Chur / Zürich

\section{Hermann Bruns \\ Neoklassische Umweltökonomie auf Irrwegen \\ Eine exemplarische Untersuchung der \\ neoklassischen Methode und ihrer \\ geistesgeschichtlichen Hintergründe \\ 28 DM, 159 Seiten, ISBN 3-89518-055-6}

Gut verständlich und fundiert kritisiert Bruns die traditionelle Umweltökonomie und kommt zu einem vernichtenden Fazit: Sie trägt nichts zur Analyse, geschweige denn zur Bewältigung der drängendsten Umweltprobleme bei.

\section{Hans G. Nutzinger (Hrsg.) \\ Nachhaltige Wirtschaftsweise und Energieversorgung}

Konzepte, Bedingungen, Ansatzpunkte

39,80 DM, 256 Seiten, ISBN 3-89518-023-8

"Substainability" - ein Begriff, dessen Unschärfe seine inflationäre und vor allem mißbräuchliche Verwendung begünstigt. Dieses Buch konkretisiert das Konzept der Nachhaltigkeit und zeigt am Beispiel der Energieversorgung, welche Schritte in Richtung einer nachhaltigen Wirtschaftsweise getan werden können und müssen.
Eberhard Fees

Haftungsregeln für multikausale Umweltschäden

Eine ökonomische Analyse des

Umwelthaftungsgesetzes - unter besonderer

Berücksichtigung multikausaler

Schadensverursachung

59 DM, 235 Seiten, ISBN 3-89518-044-0

Eine detaillierte Analyse und Beurteilung des Umwelthaftungsgesetzes vom 1.1.1991 aus ökonomischer Sicht.

Wolfgang Benkert, Jürgen Bunde

und Bernd Hansjürgens (Hrsg.)

Wo bleiben die Umweltabgaben?

Erfahrungen, Hindernisse, neue Ansätze

36,80 DM, 262 Seiten, ISBN 3-89518-024-6

Alle scheinen sich einig zu sein: Umweltabgaben sind ein geeignetes Instrument zur Reduzierung von Umweltbelastungen. Warum gelingt dennoch ihre Einführung nicht? Wie können die politischen Erfolgsaussichten von Umweltabgaben erhöht werden, ohne daß ihre ökologische Wirksamkeit leidet? Dieses Buch gibt Antworten aus ökonomischen, verwaltungswissenschaftlichen und politischen Perspektiven. 
(c) 20I0 Authors; licensee IÖW and oekom verlag. This is an article distributed under the terms of the Creative Commons Attribution Non-Commercial No Derivates License (http://creativecommons.org/licenses/by-nc-nd/3.o/), which permits unrestricted use, distribution, and reproduction in any medium, provided the original work is properly cited. 